\title{
Meningitis neumocócica: experiencia de 12 años en un hospital pediátrico, previa a la inmunización universal con vacuna conjugada Pneumococcal meningitis: A 12 year experience in a children's hospital prior to the universal immunization with a conjugate vaccine
}

\author{
Dra. Griselda Berberian ${ }^{a}$, Dra. M. Guadalupe Pérez ${ }^{a}$, Dra. Carolina Epelbaum ${ }^{a}$, \\ Bqca. María del Carmen Ceinos ${ }^{b}$, Dr. Horacio Lopardo y Dra. María Teresa Rosanova ${ }^{a}$
}

\section{RESUMEN}

Introducción. La meningitis por Streptococcus pneumoniae provoca frecuentemente elevada morbimortalidad.Elobjetivodel presenteestudio fue identificar las características epidemiológicas y clínicas, la susceptibilidad antibiótica y la evolución de los niños con meningitis por neumococo antes de la introducción de la vacuna en Argentina.

Métodos. Se incluyeron pacientes menores de 18 años internados en el Hospital J. P. Garrahan entre 1999 y 2010. Se revisaron los registros del laboratorio de microbiología y las historias clínicas de los niños.

Resultados. Se identificaron 111 niños con meningitis por S. pneumoniae. En el período 1999-2002, hubo 40 casos, 35 en 2003-2006 y 36 en 2007-2010. La media de edad fue 7 meses (r: 1-191). Eran inmunocompetentes 104 pacientes $(94 \%)$. Solo 20 pacientes $(18 \%)$ tenían enfermedad de base. La presentación clínica más frecuente fue el compromiso neurológico en 80 pacientes $(75 \%$ ) y la sepsis en 59 pacientes $(53 \%)$. Requirieron admisión en terapia intensiva 49 pacientes (44\%). Otro foco clínico de infección estuvo presente en 24 pacientes $(22 \%)$; en la mitad de ellos, neumonía. El cultivo de líquido cefalorraquídeo fue positivo en 103 pacientes (93\%) y los hemocultivos en 88 pacientes $(79 \%)$. Se identificó resistencia a la penicilina en 15\% de los casos y en el $5 \%$ resistencia a cefotaxima. La resistencia antibiótica disminuyó a lo largo de los años. Presentaron complicaciones 56 pacientes $(50 \%)$, y 11 pacientes $(10 \%)$ fallecieron por la infección.

Conclusión. La resistencia de S. pneumoniae a los antimicrobianos disminuyó a lo largo de los años. Es importante mantener la vigilancia epidemiológica para evaluar el impacto de la vacunación en Argentina.

Palabras clave: Streptococcus pneumoniae, meningitis, pediatría, resistencia antibiótica.

http:/ /dx.doi.org/10.5546/aap.2014.332

\section{INTRODUCCIÓN}

La meningitis bacteriana representa una causa importante de morbimortalidad en pediatría. En los países desarrollados, se estima que entre el 10 y el 20\% de los niños con meningitis bacteriana fallecen por la enfermedad. ${ }^{1}$ Estas cifras llegan hasta un $50 \%$ en los países en vías de desarrollo y gran parte de los sobrevivientes quedan con secuelas. ${ }^{2}$

Los principales agentes etiológicos de la meningitis bacteriana en niños son Streptococcus pneumoniae, Neisseria meningitidis y Haemophilus influenzae tipo b. ${ }^{3} \mathrm{Su}$ frecuencia varía de acuerdo con las características epidemiológicas, demográficas y con las inmunizaciones implementadas en cada región.

En Argentina, según datos del Ministerio de Salud de la Nación, el S. pneumoniae seguía siendo el primer agente etiológico causante de meningitis bacteriana en el año 2012. ${ }^{4}$ Estudios poblacionales recientes han evaluado el impacto de la meningitis neumocócica en nuestro país y han encontrado el antecedente de meningitis por S. pneumoniae en el $27 \%$ de los pacientes con hipoacusia, $15 \%$ de los que presentaban sordera profunda y $17 \%$ de los que tenían retardo mental. ${ }^{5}$

La sensibilidad del S. pneumoniae a los antimicrobianos se ha ido modificando en los últimos años. Por ello resulta necesario el conocimiento actualizado de la sensibilidad antibiótica en la población para poder indicar un tratamiento antibiótico empírico adecuado ante la sospecha de meningitis neumocócica.

El objetivo de este trabajo fue evaluar las características epidemiológicas, clínicas y evolutivas 
de los niños con meningitis neumocócica y determinar la evolución de la sensibilidad a los antimicrobianos previa a la introducción de la vacuna conjugada en el calendario nacional de vacunación en el año 2011.

\section{MATERIAL Y MÉTODOS}

Se realizó un estudio retrospectivo, observacional. Se incluyeron todos los pacientes menores de 18 años internados en el hospital con diagnóstico de meningitis por S. pneumoniae desde el 1 de enero de 1999 al 31 de diciembre de 2010. Los criterios de inclusión fueron niños menores de 18 años internados con diagnóstico de meningitis o meningoencefalitis agudas e identificación microbiológica de S. pneumoniae a partir de muestras de líquido cefalorraquídeo (LCR) y/o hemocultivos. Se excluyeron los pacientes con diagnóstico de meningitis secundaria, derivación ventrículo-peritoneal o posquirúrgica y los que presentaron solo la reacción de aglutinación con látex positiva para neumococo sin aislamiento microbiológico.

Los datos se obtuvieron mediante la revisión de las historias clínicas y estudios microbiológicos. Se registraron las características demográficas, clínicas y de evolución, además de la sensibilidad a los antimicrobianos en cada uno de los casos. Se evaluó la mortalidad a los 30 días de internación y las secuelas neurológicas al momento del alta hospitalaria. El estudio se realizó garantizando los principios éticos de la investigación en salud.

Aspectos microbiológicos: el S. pneumoniae fue caracterizado por reconocimiento de su morfología típica en la coloración de Gram y por la presentación de alfa hemólisis en placas de Agar Columbia con 5\% de sangre de carnero. Se realizó su identificación final por inhibición de crecimiento con discos de optoquina (Laboratorios Britania Argentina), solubilidad en sales biliares y aglutinación con partículas de látex (Directigen, Becton Dickinson, Cockeysville, Maryland, EE.UU.).

En todas las cepas, se determinó la sensibilidad a la penicilina con discos de $1 \mu \mathrm{g}$ de oxacilina, según recomendaciones del Clinical and Laboratory Standards Institute (CLSI) de los Estados Unidos. Se determinó la concentración inhibitoria mínima (CIM) a la penicilina y a las cefalosporinas de tercera generación por el método de Etest (Biomérieux Argentina). La sensibilidad a la penicilina y a las cefalosporinas de tercera generación se determinó de acuerdo con los puntos de corte sugeridos por el CLSI para meningitis: ${ }^{6}$ sensibilidad CIM $\leq 0,06 \mu \mathrm{g} /$ $\mathrm{ml}$ y resistencia $\mathrm{CIM} \geq 0,12 \mu \mathrm{g} / \mathrm{ml}$. Para las cefalosporinas de tercera generación, se consideró que los neumococos eran sensibles si presentaban una $\mathrm{CIM} \leq 0,5 \mu \mathrm{g} / \mathrm{ml}$ y resistentes si tenían una $\mathrm{CIM} \geq 2 \mu \mathrm{g} / \mathrm{ml}$. Los neumococos con CIM=1 $\mu \mathrm{g} /$ $\mathrm{ml}$ fueron considerados de sensibilidad intermedia.

\section{RESULTADOS}

Entre el 1 de enero de 1999 y el 31 de diciembre de 2010, se incluyeron 111 niños con cuadro clínico compatible con meningitis e identificación de S. pneumoniae en LCR y/o hemocultivos.

La media de edad de los pacientes (p) fue 7 meses (r: 1-191 m); 58 pacientes (54\%) fueron del sexo femenino. Eran inmunocompetentes 104 pacientes $(94 \%)$. Solo 7 pacientes $(6 \%)$ tenían algún tipo de inmunocompromiso: síndrome nefrótico en $4 \mathrm{p}$; HIV en $1 \mathrm{p}$; asplenia en $1 \mathrm{p}$; LMA en 1 paciente.

Tenían alguna enfermedad de base 23 pacientes $(18 \%)$. Las más frecuentes fueron cardiopatía congénita en 7 pacientes $(6 \%)$, enfermedades del sistema nervioso central en 3 pacientes $(3 \%)$ y malformaciones congénitas sin cardiopatía en 3 pacientes (3\%).

Solo 2 pacientes $(2 \%)$ tenían el antecedente de vacunación para neumococo. La media de evolución previa al diagnóstico fue de 2 días (rango: 1-7).

Requirieron internación en terapia intensiva 49 pacientes $(44 \%)$, con una media de permanencia de 5 días; 33 pacientes (30\%) requirieron asistencia respiratoria mecánica.

Al momento del ingreso hospitalario, todos los niños presentaron fiebre, y 80 pacientes (72\%) tenían algún síntoma neurológico. En esta serie, también la alteración de la conciencia fue el signo más común. La meningitis se asoció a otro foco clínico de infección en 24 pacientes (22\%): neumonía en 12 pacientes (50\%), otitis media en 7 pacientes $(29 \%)$, celulitis periorbitaria en 2 pacientes $(8 \%)$, sinusitis en 2 pacientes $(8 \%)$, diarrea en 2 pacientes ( $8 \%$ ). Se presentaron con sepsis 59 pacientes (53\%). La media de duración de la fiebre fue de 3,5 días (r: 2-15 d).

El laboratorio de ingreso mostró un recuento de glóbulos blancos (media) de 15 900/ $\mathrm{mm}^{3}$ (r: 5-45 000) y una eritrosedimentación en la primera hora de $80 \mathrm{~mm}$ (r: 40-120).

El citoquímico del LCR presentó una media de 1335 células $/ \mathrm{mm}^{3}$ (r: 0-10 000), glucosa de $28 \mathrm{mg} / \mathrm{dl}$ (r: 0-51) y proteínas de $250 \mathrm{mg} / \mathrm{dl}$ (r: 11-967). En el 82\% (n: 91 p), el predominio fue polimorfonuclear. 
La tomografía axial computada de cerebro fue solicitada en 84 pacientes (77\%) al momento del ingreso y fue patológica en 56 pacientes (66\%).

El tratamiento empírico inicial fue adecuado en 107 pacientes (96\%). En todos los casos, se utilizaron cefalosporinas de tercera generación en el tratamiento inicial y, en 22 pacientes (20\%), además, se agregó vancomicina.

La media de duración del tratamiento antibiótico fue de 13 días (r: 1-45 d). Recibieron corticoides antes de la administración del antimicrobiano 100 pacientes (90\%).

Tuvieron alguna complicación relacionada con la meningitis 58 (52\%) niños. Las más frecuentes fueron colección subdural en 24 pacientes (46\%), hidrocefalia no comunicante en 7 pacientes $(13 \%)$, vasculitis en 3 pacientes (6\%), empiema subdural en 1 pacientes $(2 \%)$ y absceso cerebral en 1 pacientes $(2 \%)$.

La media en días de internación fue de $18 \mathrm{~d}$ (r: 1-150 d). La mortalidad evaluada a los 30 días del episodio fue del 10\% (11 p).

Presentaron secuelas neurológicas permanentes 36 pacientes (33\%): retraso madurativo en 10 pacientes (9\%), epilepsia en 6 pacientes (5\%), hemiparesia en 6 pacientes $(5 \%)$ y compromiso de pares craneanos en 4 pacientes (4\%). En el seguimiento, solo pudo evaluarse la audición en 54 pacientes y 10 (19\%) de ellos tuvieron algún grado de compromiso auditivo.

Todos tuvieron aislamiento microbiológico de S. pneumoniae: LCR en 103 pacientes (93\%) y hemocultivos en 88 pacientes (79\%). Las cepas identificadas fueron $94(85 \%)$ cepas sensibles a la penicilina y 105 (95\%) sensibles a las cefalosporinas de tercera generación.

Dividiendo el estudio en tres períodos consecutivos de 4 años cada uno (período I: 1999-2002; período II: 2003-2006; período III: 2007-2010), se observó que la resistencia a la penicilina fue disminuyendo a través de los períodos: 10/40 (25\%) en el período I, 5/35 (14\%) en el período II y $2 / 36(5 \%)$ en el período III. En cuanto a la resistencia a las cefalosporinas de tercera generación, las cepas resistentes se presentaron exclusivamente en el primer período (6/40 casos). Al igual que en el resto del mundo, no hubo identificación de S. pneumoniae resistentes a la vancomicina.

\section{DISCUSIÓN}

La enfermedad invasiva por neumococo se encuentra dentro de las principales causas de morbimortalidad en la edad pediátrica. En
Argentina, actualmente, el S. pneumoniae es el primer agente causal de bacteriemia y meningitis bacteriana en niños. ${ }^{3}$

La meningitis, junto con la sepsis, representan las formas de presentación de la enfermedad invasiva por S. pneumoniae de mayor gravedad por su alta mortalidad y la elevada tasa de secuelas asociadas. ${ }^{4}$

En la era previa a la introducción de la vacuna conjugada para neumococo, la enfermedad invasiva por S. pneumoniae era la etiología prevalente en niños menores de 2 años. ${ }^{7}$ Los casos de meningitis neumocócica se reportan en pacientes aún menores. ${ }^{8,9}$ Coincidiendo con la literatura, en la serie presentada, la media de edad fue de 7 meses.

Si bien las alteraciones inmunológicas, el antecedente de fractura de la base del cráneo y las fístulas de líquido cefalorraquídeo son factores predisponentes para la meningitis por $S$. pneumoniae, ${ }^{10}$ la mayoría de los casos se presenta en niños previamente sanos. La frecuencia de comorbilidades varía en la literatura entre un $13 \%{ }^{11}$ y un $32 \%{ }^{12}$ En el estudio presentado aquí, el $18 \%$ de los niños tenían alguna enfermedad de base.

El signo neurológico más frecuente de la meningitis por S. pneumoniae descrito en la literatura y en la serie presentada es la alteración del nivel de conciencia. ${ }^{11}$

El aumento del número de células en el LCR superior a 500 células $/ \mathrm{mm}^{3}$, la hipoglucorraquia y la hiperproteinorraquia son características constantes en las diferentes series de pacientes con meningitis por S. pneumoniae. ${ }^{9-13}$ Geiseler et al. ${ }^{13}$ estudiaron 1064 pacientes con meningitis bacteriana y solo 1,5\% tuvieron celularidad normal. En esa serie, todos los pacientes con meningitis sin pleocitosis correspondieron a meningitis por Neisseria meningitidis.

Las complicaciones y la morbilidad secundaria son uno de los principales problemas asociados a la meningitis. Algunos estudios encontraron una mayor frecuencia de complicaciones neurológicas en pacientes que presentaban S. pneumoniae como agente etiológico. ${ }^{14,15}$ Un estudio realizado en el Hospital de Niños Pedro de Elizalde de la Ciudad de Buenos Aires, que incluyó a 81 pacientes con meningitis, reportó un $60 \%$ de complicaciones en las meningitis por S. pneumoniae y un $22 \%$ por otras etiologías. ${ }^{15}$ Abate et al. ${ }^{16}$ encontraron un $42 \%$ de complicaciones en 221 pacientes con meningitis por S. pneumoniae. En el presente estudio, la mitad de los niños tuvieron alguna 
complicación asociada a la meningitis y una tercera parte desarrollaron secuelas neurológicas.

La mortalidad asociada a la meningitis neumocócica, según la bibliografía, oscila entre el 2,6\% $\%^{6}$ y el $11,4 \% .{ }^{17}$ En el estudio presentado, la mortalidad fue del $10 \%$, cifra similar a la de otras series pediátricas.

La resistencia de S. pneumoniae a los antibióticos varía según las características epidemiológicas locales, los serotipos circulantes, el uso de vacunas antineumocócicas y las políticas locales de uso de antimicrobianos. ${ }^{18}$

La evolución de la resistencia a los antibióticos en S. pneumoniae en Latinoamérica se encuentra descrita en los informes del Sistema de Redes de Vigilancia de los Agentes Bacterianos Responsables de Neumonías y Meningitis (SIREVA), cuyo objetivo es la vigilancia regional de la sensibilidad antibiótica y de los serotipos predominantes. En Argentina, durante el año 2006, se identificó un $23,3 \%$ de cepas de S. pneumoniae de meningitis resistentes a la penicilina y un $5,4 \%$ de neumococos resistentes a las cefalosporinas de tercera generación. ${ }^{19}$ Para el año 2012, la resistencia a la penicilina fue de 20,8\% y no hubo cepas resistentes a las cefalosporinas de tercera generación. ${ }^{20}$ Esto coincide con la disminución de la resistencia antibiótica en términos de CIM a lo largo del período de observación presentado en este estudio.

En países en donde se implementa en forma universal la vacunación para neumococo, se ha observado una importante disminución en el número de hospitalizaciones por meningitis ${ }^{21}$ y un aumento en la incidencia de serotipos no vacunales. ${ }^{22}$

Sobre la base de los serotipos circulantes, estudios realizados en Argentina informaron una cobertura potencial para infecciones invasivas del $73 \%{ }^{23}$ y del $82,2 \%{ }^{24}$ de las vacunas antineumocócicas conjugadas 10 y 13 valente, respectivamente. La reciente incorporación de esta vacuna al calendario nacional de inmunizaciones en Argentina plantea la necesidad de mantener la vigilancia para conocer el impacto de esta medida en la epidemiología de la enfermedad neumocócica en nuestro país. ${ }^{25}$

La elección del tratamiento antibiótico empírico para las meningitis por S. pneumoniae depende de los patrones de sensibilidad a la penicilina y a las cefalosporinas de tercera generación en cada región. De acuerdo con los resultados reportados en el presente estudio y en estudios regionales, ${ }^{19}$ las cefalosporinas de tercera generación continúan siendo el antibiótico de elección recomendado para las meningitis primarias de la comunidad en huéspedes inmunocompetentes.

La característica retrospectiva de la investigación, la dificultad en el seguimiento a largo plazo para la evaluación de secuelas y la falta de identificación en los serotipos de $S$. pneumoniae de los casos incorporados al estudio constituyen las principales debilidades del presente trabajo.

\section{CONCLUSIONES}

El número de casos de meningitis neumocócica fue similar a lo largo de los 12 años, pero la resistencia disminuyó durante el período estudiado.

El último hallazgo de S. pneumoniae resistente a las cefalosporinas de tercera generación ocurrió en el año 2001.

Es necesario mantener la vigilancia epidemiológica del número, patrones de resistencia y los serotipos circulantes de S. pneumoniae a partir de la incorporación de la vacuna antineumocócica en el calendario nacional para poder evaluar las posibles modificaciones en nuestro medio.

\section{BIBLIOGRAFÍA}

1. Swartz MN. Bacterial meningitis: a view of the past 90 years. N Engl J Med 2004;351(18):1826-8.

2. Goetghebuer T, West TE, Wermenbol V, Cadbury AL, et al. Outcome of meningitis caused by Streptococcus pneumoniae and Haemophilus influenzae type b in children in The Gambia. Trop Med Int Health 2000;5(3):207-13.

3. Rubinsky R. Infecciones por Streptococcus pneumoniae. En: Paganini H. Infectología Pediátrica. Buenos Aires: Editorial Científica Interamericana; 2007. Págs.975-85.

4. Ministerio de Salud de la Nación Argentina. Secretaría de Promoción y Programas Sanitarios. Boletín Integrado de Vigilancia. N. ${ }^{\circ} 154$ - SE 2 enero de 2013. Pág. 6. [Acceso: 14 de marzo de 2014]. Disponible en: http: / / www.msal.gov.ar/images / stories/boletines / BoletinIntegradoDeVigilanciaVersion_N154-SE2.pdf.

5. Giglio N, Micone P, Gentile A. The pharmacoeconomics of pneumococcal conjugate vaccines in Latin America. Vaccine 2011;29(Suppl 3):C35-42.

6. Clinical and Laboratory Standards Institute. Performance standards for antimicrobial susceptibility testing: TwentySecond informational supplement. CLSI document M100-S22. Vol. 32(3). Wayne, PA: Clinical and Laboratory Standards Institute; 2012. [Acceso: 17 de marzo de 2014]. Disponible en: http:/ / antimicrobianos.com.ar/ATB/wpcontent/uploads/2012/11/M100S22E.pdf.

7. Haddy RI,PerryK,ChackoCE,HeltonWB, etal.Comparison of incidence of invasive Streptococcus pneumoniae disease among children before and after introduction of conjugated pneumococcal vaccine. Pediatr Infect Dis J 2005;24(4):320-3.

8. Eskola J, Takala AK, Kela E, Pekkanen E, et al. Epidemiology of invasive pneumococcal infections in children in Finland. JAMA 1992;268(23):3323-7.

9. Usen S, Adegbola R, Mulholland K, Jaffar S, et al. 
Epidemiology of invasive pneumococcal disease in the Western Region, The Gambia. Pediatr Infect Dis J 1998;17(1):23-8.

10. Durand ML,CalderwoodSB, WeberDJ, MillerSI, etal. Acute bacterial meningitis in adults. A review of 493 episodes. $N$ Engl J Med 1993;328(1):21-8.

11. Bingen E, Levy C, Varon E, de La Rocque F, et al. Pneumococcal meningitis in the era of pneumococcal conjugate vaccine implementation. Eur J Clin Microbiol Infect Dis 2008;27(3):191-9.

12. Soult Rubio JA, Rangel Pineda C, Muñoz Sáez M, Parrilla Parrilla JS, et al. Meningitis neumocócica: características epidemiológicas, clínicas y bacteriológicas. An Esp Pediatr 2001;55(4):315-20.

13. Geiseler PJ,Nelson KE. Bacterial meningitis without clinical signs of meningeal irritation. South Med J1982;75(4):448-50.

14. Arditi M, Mason EO Jr, Bradley JS, Tan TQ, et al. Threeyear multicenter surveillance of pneumococcal meningitis in children: clinical characteristics, and outcome related to penicillin susceptibility and dexamethasone use. Pediatrics 1998;102(5):1087-97.

15. Davenport MC, Del Valle MP, Gallegos P, Kannemann $\mathrm{AL}$, Bokser VS. Meningitis bacteriana: factores de riesgo para el desarrollo de complicaciones agudas. Arch Argent Pediatr 2007;105(5):405-10.

16. Abate H, Apra E, Barros F, Cibau C, Rosaenz A. Morbimortalidad de las meningitis bacterianas infantiles, no neonatales. Arch Argent Pediatr 1999;97(5):300-5.

17. Levy $C$, Varon E, Bingen $E$, Picard $C$, et al. Méningites à pneumocoque de l'enfant en France: 832 cas de 2001 à 2007. Arch Pediatr 2008;15(Suppl 3):S111-8.

18. Castañeda E, Agudelo CI, Regueira M, Corso A, et al. Laboratory-based surveillance of Streptococcus pneumoniae invasive disease in children in 10 Latin American countries: a SIREVA II project, 2000-2005. Pediatr Infect Dis J 2009;28(9):e265-70.
19. Informe Regional de SIREVA II, 2006: datos por país y por grupos de edad sobre las características de los aislamientos de Streptococcus pneumoniae, Haemophilus influenzae y Neisseria meningitidis en procesos invasores. Washington, DC: OPS, 2008. [Acceso: 17 de marzo de 2014]. Disponible en:http://www1.paho.org/Spanish/AD/THS/EV/labs_ Sireva_II_2006.pdf.

20. Informe Regional de SIREVA II, 2012: datos por país y por grupos de edad sobre las características de los aislamientos de Streptococcus pneumoniae, Haemophilus influenzae y Neisseria meningitidis en procesos invasores. Washington, DC: OPS, 2012. [Acceso: 17 de marzo de 2014]. Disponible en:http:/ / www.paho.org/hq/index.php?option=com_co ntent\&view $=$ category\&layout $=$ blog $\& i d=3609 \&$ Itemid $=39$ $53 \&$ lang $=$ es.

21. Tsai CJ, Griffin MR, Nuorti JP, Grijalva CG. Changing epidemiology of pneumococcal meningitis after the introduction of pneumococcal conjugate vaccine in the United States. Clin Infect Dis 2008;46(11):1664-72.

22. Hsu HE, Shutt KA, Moore MR, Beall BW, et al. Effect of pneumococcal conjugate vaccine on pneumococcal meningitis. N Engl J Med 2009;360(3):244-56.

23. Reijtman V, Fossati S, Hernández C, Sommerfleck P, et al. Serotype distribution of pneumococci isolated from pediatric patients with acute otitis media and invasive infections, and potential coverage of pneumococcal conjugated vaccines. Rev Argent Microbiol 2013;45(1):27-33.

24. Pérez GM, Parra A, Casimir L, Mastroianni A, et al. Infecciones invasivas por Streptococcus pneumoniae en un hospital pediátrico de tercer nivel antes de la introducción de la vacuna conjugada. Características clínicas y serotipos involucrados. Arch Argent Pediatr 2013;111(3):202-5.

25. Ministerio de Salud de la Nación Argentina. Resolución 502/2011. [Acceso: 17 de marzo de 2014]. Disponible en http://www.msal.gov.ar/pronacei/index.php/ institucional/marco-legal/375-resolucion-5022011. 